\title{
HIV IN 2005: THE CURRENT SITUATION AND DIRECTIONS FOR THE FUTURE
}

\author{
Kerri Viney \\ NSW Public Health Officer Training Program \\ NSW Department of Health
}

\section{Kim Stewart}

AIDS and Infectious Diseases Branch

NSW Department of Health

\section{Roger Garsia}

HIV/ AIDS Unit

Royal Prince Alfred Hospital

The Bug Breakfast topic for October was 'HIV in 2005: The current situation and directions for the future'. The presenters covered the history and epidemiology of HIV, emerging HIV epidemics, clinical aspects of HIV, future treatment options and NSW Health's NSW HIV/AIDS Strategy 2006-2009.

\section{EPIDEMIOLOGY OF HIV}

The World Health Organization estimates that in 2004 there were 39.4 million people living with HIV (including 4.9 million people who were newly infected) and 3.1 million deaths due to AIDS. ${ }^{1}$ More than 95 per cent of new HIV infections occurred in low and middle-income countries, with people aged between 15 and 49 years accounting for 86 per cent of these. ${ }^{1}$ In 2004 , the global prevalence of HIV ranged from 0.1 per cent in East Asia to 7.4 per cent in Sub Saharan Africa. ${ }^{1}$

In Australia the adult prevalence of HIV is 0.1 per cent. ${ }^{2}$ Nationally, the number of HIV notifications peaked in 1985 with 1770 notifications while AIDS notifications peaked in 1994 with 952. ${ }^{3}$ Although notifications have declined since this time, there has been a recent increase in HIV notifications: rising from 656 in 2000 to 818 in 2004. ${ }^{3}$ AIDS diagnoses have increased marginally from 206 in 1999 to 239 in $2004 .^{3}$ The highest notification rate for HIV in 2004 was in NSW (5.9 cases per 100,000), followed by Victoria (4.3 cases per 100,000) and Queensland (3.4 cases per 100,000$){ }^{3}$

In NSW, the number of HIV notifications peaked in 1987 and AIDS notifications in 1994. ${ }^{4}$ After 1987 there was a continual decline in HIV notifications until 2002-2003, when a slight increase was observed. However, a subsequent decline was reported in 2004. AIDS notifications also followed this pattern. ${ }^{4}$ Figure 1 displays the HIV and AIDS notifications in NSW for 1986-2004.

In NSW, 'men who have sex with men' remains the largest exposure category for patients with HIV infection, and in 2004 accounted for 62 per cent of total cases. Heterosexual

\footnotetext{
*Bug Breakfast is the name given to a monthly series of hour-long breakfast seminars on communicable diseases delivered by the NSW Department of Health's Division of Population Health.
}

exposure accounted for 18 per cent of all cases, and injecting drug use for 4 per cent. ${ }^{4}$

\section{THE NSW HIVIAIDS STRATEGY 2006-2009}

The NSW Department of Health is in the process of finalising the NSW HIV/AIDS Strategy 2006-2009. The Strategy will provide the overarching framework and priorities for HIV/AIDS prevention, treatment, care and support, research, and workforce development in NSW.

The Strategy identifies six targets for the NSW HIV/AIDS program for the period of 2006-2009; these include:

- to reduce newly acquired HIV infections by 25 per cent by 2009

- to achieve annual reductions in the notification rates of gonorrhoea, infectious syphilis and chlamydia infections among priority populations

- to reduce the physical and psychological disorders and associated disabilities in people living with HIV/ AIDS

- to decrease the number of late diagnoses of HIV infection by 25 per cent by 2009

- to achieve successive annual reductions in AIDS-related deaths by 2009

- to increase the number and distribution of clinicians authorised to prescribe highly specialised oral HIV drugs under section 100 of the National Health Act, 1953 across NSW

- to increase the number of general practitioners involved in HIV care by 20 per cent by 2009 .

The introduction of these targets establishes measurable health outcomes that decision makers can use to monitor the impact of the NSW HIV/AIDS program and inform the allocation of resources.

The challenges that face NSW in the future include:

- safeguarding gains in HIV prevention among injecting drug users, Aboriginal people and sex workers

- reducing HIV infections among inner city gay men

- addressing synergy between HIV and sexually transmissible infections (on the basis of research that shows that some sexually transmissible infections can facilitate HIV transmission)

- reducing new HIV infections and improving early diagnosis.

\section{HIV TREATMENT}

Combining multiple drugs in treatment regimens known as Highly Active Anti-Retroviral Therapy (HAART) results in large reductions in HIV viral load in previously untreated patients. This has revolutionized treatment outcomes, with 
FIGURE 1

NEW HIV AND AIDS NOTIFICATIONS BY YEAR OF DIAGNOSIS IN NEW SOUTH WALES, 1986-2004

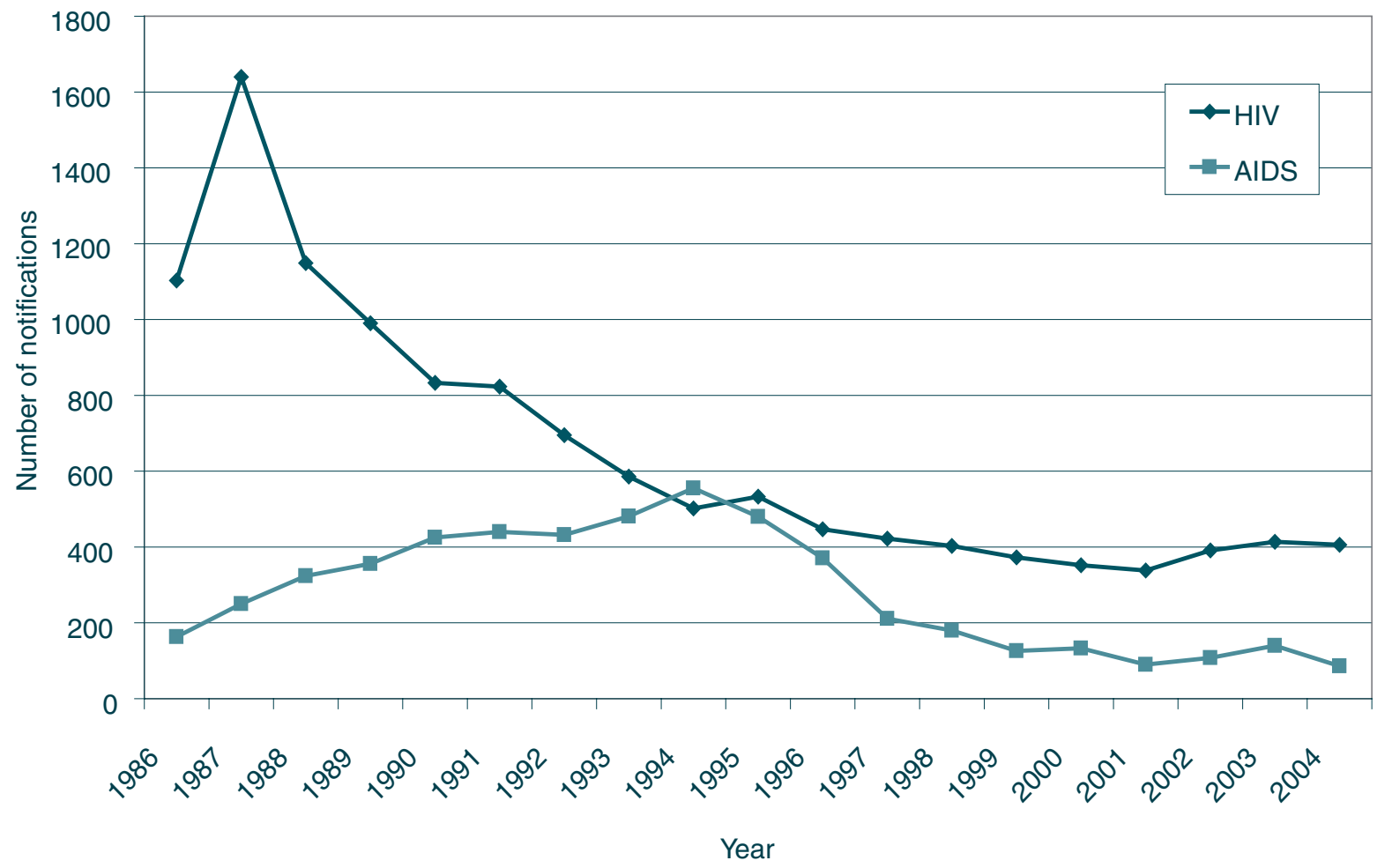

many patients continuing to achieve viral suppression for more than seven years after initiation of HAART.

However, HAART is associated with an increased rate of cardiovascular morbidity, proportionate to the duration of therapy. This has prompted enhanced efforts to control cardiovascular risk factors in HIV patients, in particular the management of lipid levels, smoking, diabetes and hypertension. More recently licensed antiretroviral drugs have demonstrated variable impact on lipid levels and may provide advantages over earlier agents.

The emergence of resistance to HIV drugs results from mutation. Initially the virus experiences a change in the genetic code and this results in structural change to the target of the drug, usually a critical enzyme or outer component of the HIV virus.

To combat drug resistance, scientists have attempted to develop drugs that target other stages of the viral life cycle. Some of these strategies look promising. Studies of new protease inhibitors and non-nucleoside reverse transcriptase inhibitors suggest that they may be beneficial in patients who have developed resistance.

Studies to develop effective vaccines are underway in many countries, including Australia; however, it is likely to be many years before an effective vaccine will be available. In addition, controversy surrounds the level of vaccine efficacy that would be needed to justify a vaccination campaign. If the protection rate were only modest, there would be a risk of producing a false sense of protection among those who were vaccinated.

\section{CONCLUSION}

HIV continues to be a problem of major public health importance globally and in NSW, where new notifications increased in the early 2000s. NSW Health has developed The NSW HIV/AIDS Strategy 2006-2009 to address this problem. While great advances have been made in treatment in the past five years, a vaccine remains some way off.

\section{REFERENCES}

1. UNAIDS: Joint United Nations Programme on HIV/AIDS. 2004 Report on the global AIDS epidemic. Geneva: UNAIDS, 2004.

2. UNAIDS: Joint United Nations Programme on HIV/AIDS / World Health Organization. AIDS epidemic update 2004. Geneva: UNAIDS/ WHO, 2004.

3. National Centre for HIV Epidemiology and Clinical Research. 2005 Annual surveillance report HIV/ AIDS, viral hepatitis, and sexually transmissible infections in Australia. Canberra, National Centre for HIV Epidemiology and Clinical Research, University of New South Wales, and Australian Institute of Health and Welfare, 2005.

4. NSW Health. Notifiable Diseases Database. NSW Health, Sydney, 2005. 